\title{
Late-time oscillatory behaviour for self-gravitating scalar fields
}

\author{
Alan D Rendall \\ Max-Planck-Institut für Gravitationsphysik, Albert-Einstein-Institut, Am Mühlenberg 1, \\ 14476 Golm, Germany
}

Received 28 November 2006, in final form 12 December 2006

Published 5 January 2007

Online at stacks.iop.org/CQG/24/667

\begin{abstract}
This paper investigates the late-time behaviour of certain cosmological models where oscillations play an essential role. Rigorous results are proved on the asymptotics of homogeneous and isotropic spacetimes with a linear massive scalar field as source. Various generalizations are obtained for nonlinear massive scalar fields, $k$-essence models and $f(R)$ gravity. The effect of adding ordinary matter is discussed as is the case of nonlinear scalar fields whose potential has a degenerate zero.
\end{abstract}

PACS numbers: 98.80.-k, 04.20.- $\mathrm{q}$

\section{Introduction}

Scalar fields are important in cosmology as a mechanism for producing models with accelerated expansion, both in the very early universe (inflation) and in more recent epochs. The mathematical properties of spatially homogeneous solutions of the Einstein equations coupled with different types of scalar field and ordinary matter have been studied in a number of papers [3-5, 9-11] in the case that there is accelerated expansion in the whole future of some late time. A corresponding analysis of situations where the accelerated expansion is only temporary, being succeeded by decelerated expansion, has not yet been carried out. One reason for this is that, in contrast to what is found for models with continuing accelerated expansion, the behaviour of the simplest models, which are homogeneous, isotropic and spatially flat, does not carry over to more general homogeneous models. In this paper the very simplest case of this type, the massive linear scalar field, is analysed. Then a number of other cases showing similar late-time behaviour are discussed.

Consider solutions of the Einstein equations coupled to a massive scalar field which are homogeneous, isotropic and spatially flat. The standard picture of the dynamics of these solutions is as follows [1]. (See also the earlier papers [14, 15].) The solutions exhibit accelerated expansion at intermediate times and eventually enter a phase where they are on average decelerated and resemble dust solutions. The main aim of this paper is to prove 
rigorous results about the late-time behaviour. Formulae for this can be found in the literature (see for instance [6]). Here a proof will be presented that these formulae do provide asymptotic expansions for all solutions in a precise sense. A curious fact, which does not seem to be mentioned in the literature, is that it is a consequence of the asymptotic expressions that each solution has infinitely many phases of accelerated expansion. The early and intermediate time behaviour will not be discussed further here but note that some rigorous results on this were obtained in [8].

A key reason why the massive scalar field is more difficult to handle than the types of the scalar field which have previously been treated mathematically is the following. When deriving asymptotic expansions in this case it is repeatedly necessary to estimate integrals with upper limit infinity which are convergent but not absolutely convergent. The convergence of the integral, which is required for the proof, results from the cancellation of positive and negative contributions. In other words, there are oscillations in the solution which continue to have a significant effect for all times.

The paper is organized as follows. In section 2 asymptotic expansions are proved for the basic example, the massive scalar field. This is generalized to a large class of nonlinear massive scalar fields in section 3. It is shown that a number of features of the asymptotics are unchanged and the expansion is derived up to the point where the first modification occurs. It is discussed briefly how these results can be applied to $f(R)$ theories of gravity. Analogous results for certain $k$-essence models are also derived. In all of this the only matter present is the scalar field. The question of the incorporation of ordinary matter such as a perfect fluid is discussed in section 4. In section 5 it is investigated what happens for a scalar field whose potential has a degenerate minimum where it vanishes. Conclusions and possible extensions of the results obtained are the subject of the last section.

\section{The massive scalar field}

This section is concerned with a massive linear scalar field $\phi$ minimally coupled to the Einstein equations under the assumptions of a spatially flat Friedmann model and the absence of any other matter fields. To simplify the computations it is assumed that the scalar field has unit mass. The scale factor is denoted by $a$ and the Hubble parameter is given by $H=\dot{a} / a$. The equation of motion of the scalar field is $\ddot{\phi}+3 H \dot{\phi}+\phi=0$ and the Hamiltonian constraint is $H^{2}=\frac{4 \pi}{3}\left(\dot{\phi}^{2}+\phi^{2}\right)$. The evolution equation for $H$ is $\dot{H}=-4 \pi \dot{\phi}^{2}$. If $H$ is zero at any time $t_{1}$ then the initial data for the scalar field at time $t_{1}$ vanish. Thus, the scalar field vanishes at all times as does $H$. This is just a flat space and so from now on it will be assumed that $H$ never vanishes. It must have a constant sign, and this sign is chosen to be positive corresponding to an expanding model. The equation of motion of $\phi$ can be rewritten as

$$
\ddot{\phi}+\sqrt{12 \pi}\left(\dot{\phi}^{2}+\phi^{2}\right)^{1 / 2} \dot{\phi}+\phi=0 .
$$

The function $H$ cannot increase and so it must tend to a limit $H_{0}$ as $t \rightarrow \infty$. It will now be shown that $H_{0}$ vanishes. Assume that $H_{0}>0$ with the aim of deriving a contradiction. The functions $\phi$ and $\dot{\phi}$ are both bounded as is $H$. From the equation of motion it follows that the same is true for $\ddot{\phi}$. Differentiating the equation repeatedly with respect to time shows that higher derivatives of $\phi$ are also bounded. Let $\left\{t_{n}\right\}$ be a sequence tending to infinity and let $\phi_{n}(t)=\phi\left(t+t_{n}\right)$ and $H_{n}(t)=H\left(t+t_{n}\right)$. Using the bounds already listed, the Arzela-Ascoli theorem [13] can be applied. This implies that, after passing to a subsequence, $\phi_{n}(t)$ converges uniformly on compact sets to a limit $\phi_{\infty}(t)$. Moreover, the first and second derivatives of $\phi_{n}$ converge to the corresponding derivatives of $\phi_{\infty}$. The sequence $H_{n}$ converges uniformly to 
the constant value $H_{0}$. With this information it is possible to pass to the limit in equation (1) to obtain

$$
\ddot{\phi}_{\infty}+3 H_{0} \dot{\phi}_{\infty}+\phi_{\infty}=0 .
$$

From the Hamiltonian constraint it follows that $H_{0}^{2}=\frac{4 \pi}{3}\left(\dot{\phi}_{\infty}^{2}+\phi_{\infty}^{2}\right)$. Differentiating this relation with respect to $t$ and substituting (2) into the result shows that $\dot{\phi}_{\infty}=0$. It follows that $\ddot{\phi}_{\infty}=0$. Then using (2) again shows that $\phi_{\infty}=0$ and this leads to a contradiction to the assumption that $H_{0} \neq 0$. Thus, in fact $H(t) \rightarrow 0$ as $t \rightarrow \infty$.

Next more detailed asymptotics will be obtained. The pair

$$
\left(\phi /\left(\dot{\phi}^{2}+\phi^{2}\right)^{1 / 2}, \dot{\phi} /\left(\dot{\phi}^{2}+\phi^{2}\right)^{1 / 2}\right)
$$

defines a function of $t$ with values in the unit circle. Thus, it is possible to define a realvalued function $\theta(t)$ whose projection to the circle under identification modulo $2 \pi$ is the given function with values in the circle. The function $\theta$ is unique up to shifts of its argument by integer multiples of $2 \pi$. Let $r=\left(\dot{\phi}^{2}+\phi^{2}\right)^{1 / 2}$. Then the following equations can be derived:

$$
\begin{aligned}
& \dot{r}=-\sqrt{12 \pi} r^{2} \sin ^{2} \theta \\
& \dot{\theta}=-1-\sqrt{12 \pi} r \sin \theta \cos \theta .
\end{aligned}
$$

Given $\epsilon>0$ there exists a time $t_{1}$ such that $\sqrt{12 \pi} r \leqslant \epsilon$ for $t \geqslant t_{1}$. It follows that on that interval $|\dot{\theta}+1| \leqslant \epsilon$. This means that

$$
\theta_{1}-(1+\epsilon)\left(t-t_{1}\right) \leqslant \theta(t) \leqslant \theta_{1}-(1-\epsilon)\left(t-t_{1}\right),
$$

where $\theta_{1}=\theta\left(t_{1}\right)$. The evolution equation for $r$ can be rewritten in the form

$$
r^{-1}(t)=r^{-1}\left(t_{1}\right)+\sqrt{3 \pi}\left[\left(t-t_{1}\right)+\int_{\theta(t)}^{\theta_{1}} \cos 2 \theta^{\prime} \dot{\theta}^{-1}\left(\theta^{\prime}\right) \mathrm{d} \theta^{\prime}\right] .
$$

Suppose that $\epsilon<1$. Let $k$ be the largest integer such that $\theta_{1}-k \pi \geqslant \theta(t)$. Then

$$
\left|\int_{\theta(t)}^{\theta_{1}-k \pi} \cos 2 \theta^{\prime} \dot{\theta}^{-1}\left(\theta^{\prime}\right) \mathrm{d} \theta^{\prime}\right| \leqslant \pi(1-\epsilon)^{-1} .
$$

On the other hand,

$$
\begin{aligned}
\left|\int_{\theta_{1}-k \pi}^{\theta_{1}} \cos 2 \theta^{\prime} \dot{\theta}^{-1}\left(\theta^{\prime}\right) \mathrm{d} \theta^{\prime}\right| & =\left|\int_{\theta_{1}-k \pi}^{\theta_{1}} \cos 2 \theta^{\prime}\left[\dot{\theta}^{-1}\left(\theta^{\prime}\right)+1\right] \mathrm{d} \theta^{\prime}\right| \\
& \leqslant k \pi \epsilon(1-\epsilon)^{-1} \\
& \leqslant \epsilon(1+\epsilon)(1-\epsilon)^{-1}\left(t-t_{1}\right) .
\end{aligned}
$$

Thus, the integral on the right-hand side of (6) is bounded in modulus by $(1-\epsilon)^{-1}(\pi+$ $\left.\epsilon(1+\epsilon)\left(t-t_{1}\right)\right)$. Putting this information into (6) shows that

$r^{-1}(t) \geqslant r^{-1}\left(t_{1}\right)+\sqrt{3 \pi}\left\{-(1-\epsilon)^{-1} \pi+\left[1-\epsilon(1+\epsilon)(1-\epsilon)^{-1}\right]\left(t-t_{1}\right)\right\}$.

As a consequence, $r^{-1}$ grows linearly with $t$ and $r(t)=O\left(t^{-1}\right)$. The evolution equation for $\theta$ then implies that $\dot{\theta}+1=O\left(t^{-1}\right)$ and that

$$
\theta(t)=-t+O(\log t) .
$$

Putting the improved estimate for $\dot{\theta}$ back into (6) shows that

$$
r(t)=\frac{1}{\sqrt{3 \pi} t}+O\left(t^{-2} \log t\right) .
$$


Translating this to the original variables it can be seen that the leading-order contribution to $H$ is $2 / 3 t$ and that the scale factor behaves asymptotically like $t^{2 / 3}$. Thus, the late-time dynamics is similar to that of a model with dust. To go further it is necessary to obtain an improved estimate for $\theta$. Note that

$$
\frac{\mathrm{d}}{\mathrm{d} t}(r \cos 2 \theta)=\dot{r} \cos 2 \theta-2 r \sin 2 \theta \dot{\theta}=2 r \sin 2 \theta+O\left(t^{-2}\right) .
$$

Integrating the equation for $\dot{\theta}$ and using the above relation gives

$$
\theta=-t+C+O\left(t^{-1}\right)
$$

for a constant $C$. This constant can be eliminated by choosing an appropriate origin for $t$. Then $\theta=-t+O\left(t^{-1}\right)$.

Consider now the asymptotic behaviour of the scalar field:

$$
\begin{aligned}
\cos (\theta(t)) & =\cos t \cos (\theta(t)+t)+\sin t \sin (\theta(t)+t) \\
& =\cos t+O\left(t^{-1}\right) .
\end{aligned}
$$

Hence

$$
\phi(t)=\frac{\cos t}{\sqrt{3 \pi} t}+O\left(t^{-2} \log t\right)
$$

Similarly

$$
\dot{\phi}(t)=\frac{\sin t}{\sqrt{3 \pi} t}+O\left(t^{-2} \log t\right) .
$$

Putting the expressions for $\sin \theta$ and $\cos \theta$ into the equation for $\dot{\theta}$ gives

$$
\dot{\theta}=-1-t^{-1} \sin 2 t+O\left(t^{-2} \log t\right) .
$$

This can now be substituted into (12) to get

$\frac{\mathrm{d}}{\mathrm{d} t}(r \cos 2 \theta)=2 r \sin 2 \theta-\frac{2}{\sqrt{3 \pi}} t^{-2} \sin ^{2} t \cos 2 t+\frac{2}{\sqrt{3 \pi}} t^{-2} \sin ^{2} 2 t+O\left(t^{-3} \log t\right)$.

The following lemma is useful for estimating the integral of oscillatory quantities. It is proved by integrating by parts twice.

Lemma 1. Let $F$ be a smooth periodic function with mean zero, $f=F^{\prime}$ and $k$ a positive real number. Then

$$
\int_{1}^{T} f(t) t^{-k} \mathrm{~d} t=F(T) T^{-k}+C+O\left(T^{-k-1}\right)
$$

for a constant $C$.

Integrating (18), applying lemma 1 and noting that due to the information already obtained the integration constant must vanish, it follows that

$$
\theta(t)=-t-\frac{3+2 \cos 2 t}{4 t}+O\left(t^{-2} \log t\right)
$$

Putting the information now available into the equation for the time derivative of $r^{-1}$ gives

$$
\frac{\mathrm{d}}{\mathrm{d} t}\left(r^{-1}\right)=\sqrt{3 \pi}\left(1-\cos 2 t+\sin 2 t\left(\frac{3+2 \cos 2 t}{2 t}\right)+O\left(t^{-2} \log t\right)\right)
$$

and after integration

$$
r^{-1}=\sqrt{3 \pi}\left[\left(t-t_{1}\right)-\frac{\sin 2 t}{2}\right]+O\left(t^{-1} \log t\right)
$$


for a constant $t_{1}$. It follows that

$$
H=\frac{2}{3\left(t-t_{1}\right)}\left[1+\frac{\sin 2 t}{2 t}+O\left(t^{-2} \log t\right)\right] .
$$

Note that the constant $t_{1}$ in the leading term in the expression for $H$ could be got rid of by a translation in $t$ at the expense of introducing a similar constant elsewhere. The expressions for the scalar field can now be improved to give

$\phi(t)=\frac{1}{\sqrt{3 \pi}\left(t-t_{1}\right)}\left[\cos t-\sin t\left(\frac{3+2 \cos 2 t}{4 t}\right)\right]\left[1+\frac{\sin 2 t}{2 t}\right]+O\left(t^{-3} \log t\right)$

and

$\dot{\phi}(t)=\frac{1}{\sqrt{3 \pi}\left(t-t_{1}\right)}\left[-\sin t-\cos t\left(\frac{3+2 \cos 2 t}{4 t}\right)\right]\left[1+\frac{\sin 2 t}{2 t}\right]+O\left(t^{-3} \log t\right)$.

The first of these may be compared with equation (5.45) on p 240 of [6].

Since the scale factor grows slower than linearly the expansion is on average decelerated. On the other hand, $\ddot{a}=\frac{4 \pi}{3}\left(\phi^{2}-2 \dot{\phi}^{2}\right)$ and so there are infinitely many intervals on which $\ddot{a}>0$.

\section{Nonlinear scalar fields}

The aim of this section is to investigate to what extent the results for the massive linear scalar field can be extended to the case of a potential of the form $V(\phi)=\phi^{2} / 2+W(\phi)$, where $W$ is smooth and $W(\phi)=O\left(\phi^{3}\right)$. The equation of motion for the scalar field is

$$
\ddot{\phi}+\sqrt{12 \pi}\left(\dot{\phi}^{2}+\phi^{2}+2 W(\phi)\right)^{1 / 2} \dot{\phi}+\phi+W^{\prime}(\phi)=0 .
$$

The evolution equation for $H$ is unchanged. It can be concluded that $H$ tends to a limit $H_{0}$ as $t \rightarrow \infty$. To constrain the value of $H_{0}$ a compactness argument can be used as before, leading to the limiting equation

$$
\ddot{\phi}_{\infty}+3 H_{0} \dot{\phi}_{\infty}+\phi_{\infty}+W^{\prime}\left(\phi_{\infty}\right)=0
$$

with $H_{0}^{2}=\frac{4 \pi}{3}\left(\dot{\phi}_{\infty}^{2}+\phi_{\infty}^{2}+2 W\left(\phi_{\infty}\right)\right)$. It follows that $\dot{\phi}_{\infty}=0$. Assume now that $V$ has no critical points other than the origin in an interval $\left[-\phi_{1}, \phi_{1}\right]$ and that the initial value of $H$ is smaller than $\sqrt{\frac{4 \pi}{3}} \phi_{1}$ so that $\phi$ remains in that interval. Then it follows that $\phi_{\infty}=0$ and this gives a contradiction. Thus, in fact $H_{0}=0$. As in the linear case polar coordinates $(r, \theta)$ can be introduced in the $(\phi, \dot{\phi})$ plane. In these variables the equations read

$\dot{r}=-\sqrt{12 \pi} r^{2} \sin ^{2} \theta\left(1+2 r^{-2} W(r \cos \theta)\right)^{1 / 2}-\sin \theta W^{\prime}(r \cos \theta)$

$\dot{\theta}=-1-\sqrt{12 \pi} r \sin \theta \cos \theta\left(1+2 r^{-2} W(r \cos \theta)\right)^{1 / 2}-r^{-1} W^{\prime}(r \cos \theta) \cos \theta$.

Note that $\dot{\theta}+1=O(r)$ so that it can be concluded as in the case $W=0$ that $\theta$ grows linearly with $t$. The equation for $\dot{r}$ can be rearranged to give

$$
\frac{\mathrm{d}}{\mathrm{d} t}\left(r^{-1}\right)=\sqrt{12 \pi} \sin ^{2} \theta\left(1+2 r^{-2} W(r \cos \theta)\right)^{1 / 2}+\sin \theta r^{-2} W^{\prime}(r \cos \theta) .
$$

The last term in this equation looks worrying but note that up to a remainder of order $r$ it is equal to $\frac{1}{2} W^{\prime \prime \prime}(0) \cos ^{2} \theta \sin \theta$. The last expression has mean zero and so its integral up to time $t$ is a bounded function of $t$, as can be seen by changing the variable of integration from $t$ to $\theta$. It can be concluded as in the linear case that $r^{-1}$ grows linearly with $t$ and that $r(t)=O\left(t^{-1}\right)$. 
Furthermore, $\dot{\theta}+1=O\left(t^{-1}\right)$ and (10) and (11) hold. Thus, $H(t)$ has the same leading-order behaviour as for dust in this case too.

Next further information on the asymptotics of $\theta$ will be obtained, reaching the point where the first correction coming from $W$ occurs. Note first that $\left(1+2 r^{-2} W(r \cos \theta)\right)^{1 / 2}=1+O(r)$ so that the correction coming from $W$ in the second term on the right-hand side of the equation for $\dot{\theta}$ is $O\left(t^{-2}\right)$. The last expression in (12) can be used to replace $\mathrm{d} / \mathrm{d} t(r \cos 2 \theta)$ as in the case $W=0$, and this allows the integral of $r \sin 2 \theta$ to be treated. To handle the last term in the equation for $\dot{\theta}$ note that

$r^{-1} W^{\prime}(r \cos \theta) \cos \theta=\frac{1}{6 \sqrt{3}} t^{-1} W^{\prime \prime \prime}(0) \frac{\mathrm{d}}{\mathrm{d} \theta}\left(3 \cos \theta-\sin ^{3} \theta\right)+O\left(t^{-2} \log t\right)$.

Furthermore

$$
\frac{\mathrm{d}}{\mathrm{d} \theta}\left(3 \cos \theta-\sin ^{3} \theta\right)=\frac{\mathrm{d}}{\mathrm{d} t}\left(3 \cos \theta-\sin ^{3} \theta\right)+O\left(t^{-1}\right) .
$$

Using lemma 1 this implies that $\theta=-t+O\left(t^{-1} \log t\right)$. Relations (15) and (16) follow. The first place where $W$ makes a difference in the asymptotic expansions is in the analogue of (17). It is given by

$$
\dot{\theta}=-1-t^{-1} \sin 2 t-\frac{1}{2 \sqrt{3}} t^{-1} W^{\prime \prime \prime}(0) \cos ^{3} t+O\left(t^{-2} \log t\right) .
$$

The results obtained above in the case of unit mass $m=1$ can be generalized to any positive $m$. To get the asymptotic formulae in the general case it suffices to replace $t$ by $m t$ everywhere. Theorems are obtained for the potentials $\frac{1}{2} m^{2} \phi^{2}+\lambda \phi^{4}$ and $\frac{1}{4} \lambda\left(\phi^{2}-\phi_{0}^{2}\right)^{2}$ considered in [1]. In the first case the late-time asymptotics are proved for all solutions, while in the second they are proved for all solutions starting sufficiently close to one of the two local minima of the potential.

These results can also be applied to obtain information about the $f(R)$ theories of gravity which are equivalent to the Einstein equations coupled to a nonlinear scalar field via a conformal transformation. For a discussion of this, see section 5.6 of [6]. For example, consider the theory where the Einstein-Hilbert Lagrangian is replaced by $R+\alpha R^{2}$ for a negative constant $\alpha$. The potential of the corresponding scalar field is non-negative and has a unique minimum at zero. Its second derivative at that point is $-8 \pi / 3 \alpha$. Hence the theory just developed can be applied to this case. The interval $\left[-\phi_{1}, \phi_{1}\right]$ and a bound for the value of $H$ at the initial time must be chosen appropriately. Similar arguments work for more general choices of $f(R)$. Assume that $f(0)=0, \frac{\mathrm{d} f}{\mathrm{~d} R}(0)=1$ and $\frac{\mathrm{d}^{2} f}{\mathrm{~d} R^{2}}(0)<0$. Then the corresponding scalar field has the properties which allow the results of this section to be applied to it. Since $\phi \rightarrow 0$ as $t \rightarrow \infty$ the conformal factor tends to one as $t \rightarrow \infty$ and the physical metric has the same leading-order asymptotics as the conformally rescaled one.

It may be remarked in passing that the results of [9] also have applications to determining the late-time behaviour of $f(R)$ models with continuing acceleration. If there is a positive constant $V_{0}$ such that $f\left(-2 V_{0}\right)=-V_{0}$ and $f^{\prime}\left(-2 V_{0}\right)=1$ then the corresponding potential has a minimum at zero where it takes the value $V_{0}$. Provided $f^{\prime \prime}\left(-2 V_{0}\right)$ lies in the interval $\left(-\frac{1}{2 V_{0}}, 0\right)$ then the second derivative of the potential at zero is positive and the results of [9] can be applied. Note that the case $f(R)=-R^{2}$ discussed in [16] (without symmetry assumptions on the solutions) is a borderline one since $f^{\prime \prime}\left(-2 V_{0}\right)=-\frac{1}{2 V_{0}}$. In that case the potential is constant.

Another type of generalization is to $k$ essence where the Lagrangian $X-V$ of an ordinary nonlinear scalar field, with $X=-\frac{1}{2} \nabla_{\alpha} \phi \nabla^{\alpha} \phi$, is replaced by a more general function $L(\phi, X)$. The evolution equation for $H$ with $H^{2}=\frac{8 \pi}{3}(2 X \partial L / \partial X-L)$ becomes $\dot{H}=-8 \pi X \partial L / \partial X$. 
Note that in a spatially homogeneous solution $X$ is non-negative. This Lagrangian will now be specialized to the case $L(\phi, X)=X-\frac{1}{2} \phi^{2}-W(\phi, X)$, where $W$ vanishes at the origin up to a remainder of third order. Now

$$
2 X \partial L / \partial X-L=X+\frac{1}{2} \phi^{2}-2 X \partial W / \partial X+W .
$$

It follows that there is a constant $C>0$ such that

$$
C^{-1}\left(\phi^{2}+X\right) \leqslant 2 X \partial L / \partial X-L \leqslant C\left(\phi^{2}+X\right)
$$

for $\phi$ and $X$ being small. This means in particular that when $\phi$ and $X$ are small $H$ can only be zero when both $\phi$ and $X$ are zero. As a consequence, the initial data for $\phi$ vanish so that $\phi$ vanishes everywhere. This case will be excluded from consideration and so it can be assumed as in the cases studied previously that $H>0$. Under the given assumptions on $W$ it follows that $H$ is non-increasing. Thus, if it starts small it remains small and tends to a limit $H_{0}$ as $t \rightarrow \infty$. The equation of motion of $\phi$ is

$$
\left(\frac{\partial L}{\partial X}+2 X \frac{\partial^{2} L}{\partial X^{2}}\right) \ddot{\phi}+\frac{\partial L}{\partial X}(3 H \dot{\phi})+\frac{\partial^{2} L}{\partial \phi \partial X} \dot{\phi}^{2}-\frac{\partial L}{\partial \phi}=0 .
$$

In order to repeat the compactness argument which has been used in other cases all that needs to be ensured is that the coefficient of $\ddot{\phi}$ in this equation remains bounded away from zero. If the initial value of $H$ is small enough this is the case. Passing to the limit in the evolution equation for $H$ it follows that $\dot{\phi}_{\infty}=0$. The equation of motion for $\phi_{\infty}$ implies that $\frac{\partial L}{\partial \phi}\left(\phi_{\infty}, 0\right)=0$. For $\phi_{\infty}$ being sufficiently small this means that $\phi_{\infty}=0$. Hence $H_{0}=0$.

Passing to polar coordinates in the $(\phi, \dot{\phi})$ plane the equation of motion becomes

$$
\begin{aligned}
& \dot{r}=\sin \theta[1-\left.\frac{\partial W}{\partial X}-2 X \frac{\partial^{2} W}{\partial X^{2}}\right]^{-1}\left[-\left(1-\frac{\partial W}{\partial X}\right)(3 H \dot{\phi})+\frac{\partial^{2} W}{\partial \phi \partial X} \dot{\phi}^{2}-\frac{\partial W}{\partial \phi}\right] \\
&-r \cos \theta \sin \theta\left[\frac{\partial W / \partial X+2 X \partial^{2} W / \partial X^{2}}{1-\partial W / \partial X-2 X \partial^{2} W / \partial X^{2}}\right] \\
& \dot{\theta}=-1-\cos ^{2} \theta\left[\frac{\partial W / \partial X+2 X \partial^{2} W / \partial X^{2}}{1-\partial W / \partial X-2 X \partial^{2} W / \partial X^{2}}\right]+\cos \theta\left[1-\frac{\partial W}{\partial X}-2 X \frac{\partial^{2} W}{\partial X^{2}}\right]^{-1} \\
& \times\left[-\left(1-\frac{\partial W}{\partial X}\right)(3 H \sin \theta)+\frac{\partial^{2} W}{\partial \phi \partial X} r \sin ^{2} \theta-r^{-1} \frac{\partial W}{\partial \phi}\right] .
\end{aligned}
$$

The expression $\partial W / \partial X+2 X \partial^{2} W / \partial X^{2}$, when evaluated at $\left(r \cos \theta, r^{2} \sin ^{2} \theta / 2\right)$, is $O\left(r^{2}\right)$. From this it easily follows that $\dot{\theta}+1$ is $O(r)$ and so $\theta$ grows linearly with $t$. The last term in the equation for $\dot{r}$ is $O\left(r^{3}\right)$, as is the expression $\dot{\phi}^{2} \partial^{2} W / \partial \phi \partial X$. Thus,

$$
\frac{\mathrm{d}}{\mathrm{d} t}\left(r^{-1}\right)=\sqrt{12 \pi} \sin ^{2} \theta+\sin \theta r^{-2} \frac{\partial W}{\partial \phi}+O(r) .
$$

The second term on the right-hand side of this equation can be written in the form $\frac{1}{2} \frac{\partial^{3} W}{\partial \phi^{3}}(0,0) \cos ^{2} \theta \sin \theta+O\left(r^{-1}\right)$. It can be concluded as in the case of the ordinary nonlinear scalar field that $r=O\left(t^{-1}\right)$ and that $H$ has the same leading-order behaviour as in the case of dust.

\section{Inclusion of matter}

The previous sections were concerned with the Einstein equations coupled to a scalar field (possibly nonlinear) without including other fields describing ordinary matter. In this section 
matter satisfying the dominant and strong energy conditions will be added. The energymomentum tensor is the sum of that of a scalar field and that of the other matter. There is no direct coupling between the scalar field and the ordinary matter-they interact only indirectly via their coupling to the gravitational field. The assumption of a spatially flat Friedmann model is maintained. The equation of motion of the scalar field, before substituting for $H$ using the Hamiltonian constraint, remains unchanged. The Hamiltonian constraint reads

$$
H^{2}=\frac{4 \pi}{3}\left(\dot{\phi}^{2}+\phi^{2}+2 V+2 \rho_{M}\right)
$$

where $\rho_{M}$ is the energy density of the ordinary matter. If $H$ is zero at any time $t_{1}$ the initial data for the scalar field vanish as a consequence of the weak energy condition. Then the scalar field vanishes at all times. Since the subject of interest here is the effect of a scalar field that case will be excluded. It is assumed from now on that $H$ is positive. The evolution equation for $H$ is

$$
\dot{H}=-4 \pi\left[\dot{\phi}^{2}+\rho_{M}+\frac{1}{3} \operatorname{tr} S_{M}\right]
$$

where $\operatorname{tr} S_{M}$ is the trace of the spatial projection of the energy-momentum tensor of the ordinary matter. The dominant energy condition implies that the right-hand side of (41) is non-positive so that $H(t)$ is non-increasing.

Assuming that the solution of the coupled Einstein-scalar-matter equations exists globally in the future it can be concluded that $H(t)$ tends to a limit $H_{0}$ as $t \rightarrow \infty$. The quantities $\phi, \dot{\phi}$ and $H$ are all bounded. Hence $\ddot{\phi}$ is bounded. The energy-momentum tensor of the ordinary matter is divergence-free and it follows that

$$
\mathrm{d} \rho_{M} / \mathrm{d} t+3 H\left(\rho_{M}+\frac{1}{3} \operatorname{tr} S_{M}\right)=0 .
$$

Thus, by the dominant energy condition, $\rho_{M}$ is non-increasing and converges to some limit $\rho_{\infty} \geqslant 0$. Also tr $S_{M} \leqslant 3 \rho_{M}$ and it follows that $\dot{H}$ is bounded. Differentiating the equation of motion for the scalar field shows that the third time derivative of $\phi$ is bounded. Using these facts a compactness argument can be carried out as in the case without ordinary matter. Under the same conditions it can be concluded that $\phi$ and $\dot{\phi}$ tend to zero as $t \rightarrow \infty$. Moreover, it follows from (41) that $\rho_{M}+\frac{1}{3} \operatorname{tr} S_{M} \rightarrow 0$. Hence $\operatorname{tr} S_{M} \rightarrow-3 \rho_{\infty}$ as $t \rightarrow \infty$. But then $\rho_{M}+\operatorname{tr} S_{M} \rightarrow-2 \rho_{\infty}$. If $\rho_{\infty}$ were non-zero then this would contradict the strong energy condition. Hence in fact $\rho_{\infty}=0, H_{0}=0$ and $\rho_{M}(t) \rightarrow 0$ as $t \rightarrow \infty$.

Consider for simplicity the linear case $W=0$. When the equation of motion for $\phi$ is written in polar coordinates the resulting equations are

$$
\begin{aligned}
& \dot{r}=-\sqrt{12 \pi}\left(r^{2}+2 \rho_{M}\right)^{1 / 2} r \sin ^{2} \theta \\
& \dot{\theta}=-1-\sqrt{12 \pi}\left(r^{2}+2 \rho_{M}\right)^{1 / 2} \sin \theta \cos \theta .
\end{aligned}
$$

Since $H$ tends to zero as $t \rightarrow \infty$ the argument that $\theta$ grows linearly with $t$ also works in the presence of ordinary matter. It is also true that $\dot{\theta}+1$ remains bounded away from zero. Analysis of the evolution equation for $r^{-1}$ shows that $r^{-1}$ must grow at least linearly and that $r=O\left(t^{-1}\right)$. Due to its sign the contribution of the energy density of ordinary matter can only increase this rate of decay. Unfortunately, this does not immediately give a decay rate for $H$. It would seem that to go further it would be necessary to know which of the terms $r^{2}$ or $\rho_{M}$ dominates at late times. In the case of a perfect fluid with linear equation of state $p_{M}=(\gamma-1) \rho_{M}$, heuristic considerations indicate that if $\gamma>1$ it is self-consistent to require that the fluid has a negligible effect at late times while for $2 / 3<\gamma<1$ (a model which satisfies the strong and dominant energy conditions) this is not consistent. Rigorous results on the asymptotics in these cases are not available. 


\section{Degenerate minima}

Suppose now that the linear scalar field is replaced by a nonlinear one with $V(\phi)=\phi^{2 n} / 2 n$ and no additional matter is included. Then the equation for $\dot{H}$ is unchanged and the equation of motion is

$$
\ddot{\phi}+\sqrt{12 \pi}\left(\dot{\phi}^{2}+\phi^{2 n} / n\right)^{1 / 2} \dot{\phi}+\phi^{2 n-1}=0 .
$$

As in the case $n=1$ the function $H$ is non-increasing and tends to a limit $H_{0}$. The argument that $H_{0}=0$ goes through without significant change so that $\phi$ and $\dot{\phi}$ tend to zero as $t \rightarrow \infty$. For simplicity only the special case $n=2$ will be considered in the rest of this paragraph. Passing to polar coordinates leads to the system

$$
\begin{aligned}
& \dot{r}=r \sin \theta \cos \theta\left(1-r^{2} \cos ^{2} \theta\right)-3 H r \sin ^{2} \theta \\
& \dot{\theta}=-\sin ^{2} \theta-r^{2} \cos ^{4} \theta-3 H \sin \theta \cos \theta,
\end{aligned}
$$

where

$$
H=\sqrt{\frac{4 \pi}{3}} r\left(\sin ^{2} \theta+\frac{1}{2} r^{2} \cos ^{4} \theta\right)^{1 / 2} .
$$

For the solutions being considered here $r$ never vanishes at any time. It follows that whenever $\theta=k \pi$ or $(k+1 / 2) \pi$ for $k$ an integer $\dot{\theta}<0$. Hence there are two mutually exclusive possibilities. Either $\theta(t) \rightarrow-\infty$ as $t \rightarrow \infty$ or $\theta(t)$ is eventually trapped between $\theta_{1}$ and $\theta_{1}+\pi / 2$, where $2 \theta_{1} / \pi$ is an integer. It will now be shown that the second of these cannot occur. To do this it is helpful to distinguish between the case where $\theta_{1} / \pi$ is an integer and that where $\theta_{1} / \pi+1 / 2$ is an integer. In the first case $\theta$ is monotone decreasing at late times and $\theta \rightarrow \theta_{1}$ as $t \rightarrow \infty$. For $r$ being small enough

$$
\dot{r} \geqslant r \sin \theta\left(\frac{1}{2} \cos \theta-3 H \sin \theta\right) \text {. }
$$

This implies that $\dot{r}$ is eventually positive, contradicting the fact that $H \rightarrow 0$ as $t \rightarrow \infty$. Thus, this case is ruled out and it can be assumed that $\theta_{1} / \pi+1 / 2$ is an integer. In that case $r$ is eventually monotone decreasing and it can be concluded that $r=o(1)$. For any interval of the form $\left[\theta_{1}, \theta_{2}\right]$ with $\theta_{2}<\theta_{1}+\pi / 2$, it is true that at sufficiently late times any solution for which $\theta$ lies in this interval satisfies a uniform negative upper bound on $\dot{\theta}$. Thus, $\theta \rightarrow \theta_{1}+\pi / 2$ as $t \rightarrow \infty$ and $H=o(r)$. Thus, for any $\epsilon>0$ there is a time $t_{1}$ such that for $t>t_{1}$,

$$
\begin{aligned}
-\sin ^{2} \theta-r^{2} \cos ^{4} \theta-3 H \sin \theta \cos \theta & \leqslant-\sin ^{2} \theta-r^{2} \cos ^{4} \theta+\epsilon r|\sin \theta \| \cos \theta| \\
& \leqslant-\sin ^{2} \theta\left(1-\frac{\epsilon}{2}\right)-r^{2} \cos ^{2} \theta\left(\cos ^{2} \theta-\frac{\epsilon}{2}\right) \\
& <0 .
\end{aligned}
$$

Using the evolution equation for $\theta$ this gives a contradiction. Thus, it can be concluded that in fact $\theta(t) \rightarrow-\infty$ as $t \rightarrow \infty$. It follows that there are infinitely many oscillations of the scalar field in this case too.

There is a remark on p 242 of [6] that in the case of a nonlinear scalar field with potential $V(\phi)=\phi^{2 n} / 2 n$ the solution should be approximated in some averaged sense by a perfect fluid with a linear equation of the state. A statement will now be proved which is a concrete realization of this idea. Multiplying the equation of motion for a nonlinear scalar field by $\phi$ and rearranging gives the identity

$$
\phi V^{\prime}(\phi)=\dot{\phi}^{2}-6 \pi \phi^{2} \dot{\phi}^{2}-\frac{\mathrm{d}}{\mathrm{d} t}\left(\phi \dot{\phi}+\frac{3}{2} H \phi^{2}\right) .
$$


In deriving this the equation for $\dot{H}$ has been used. The information already available concerning the solution justifies integrating this relation from some time $t$ to infinity to get

$$
\int_{t}^{\infty} \phi V^{\prime}(\phi) \mathrm{d} s=\int_{t}^{\infty} \dot{\phi}^{2}-6 \pi \dot{\phi}^{2} \phi^{2} \mathrm{~d} s-\phi(t) \dot{\phi}(t) .
$$

In the special case of the power-law potential $\phi V^{\prime}(\phi)=2 n V(\phi)$. Integrating the evolution equation for $H$ and using the fact that $H(t)$ tends to zero as $t \rightarrow \infty$ shows that

$$
\int_{t}^{\infty} \dot{\phi}^{2} \mathrm{~d} s=\frac{1}{4 \pi} H(t)
$$

Using this in (52) shows that

$$
\int_{t}^{\infty} \phi V^{\prime}(\phi) \mathrm{d} s=\left(\int_{t}^{\infty} \dot{\phi}^{2} \mathrm{~d} s\right)(1+o(1)), \quad t \rightarrow \infty .
$$

It follows that

$$
\frac{\int_{t}^{\infty} p(s) \mathrm{d} s}{\int_{t}^{\infty} \rho(s) \mathrm{d} s}=\left(\frac{n-1}{n+1}\right)(1+o(1)) .
$$

This corresponds to dust in the case $n=1$ and to radiation in the case $n=2$.

\section{Conclusions}

In this paper rigorous asymptotic expansions for the late-time asymptotics of spatially flat homogeneous and isotropic solutions of the Einstein equations coupled to a linear massive scalar field are proved. It is shown what similarities and differences there are when the quadratic potential of this model is modified by higher order corrections. The latter results apply directly to give information about the dynamics in certain $f(R)$ theories. Basic asymptotics are also obtained for $k$-essence models where the leading-order terms in the Lagrangian near the origin agree with those of a linear massive scalar field.

When ordinary matter such as a perfect fluid is added to the model some statements about the asymptotic behaviour are proved, but at a certain stage in the expansion a competition arises between the scalar field and the other matter, and the outcome of this is not settled rigorously here. In the case of a potential such as $V(\phi)=\phi^{4} / 4$ with a degenerate minimum, it is shown that the scalar field is oscillatory in the sense that it has infinitely many zeroes. A relation to the radiation fluid is derived.

As has already been indicated, the asymptotics derived in section 2 can be destroyed by the introduction of spatial curvature. When homogeneous solutions with non-vanishing spatial curvature are considered the equation of motion for $\phi$ remains the same, but the expression for $H$ given by the Hamiltonian constraint and the evolution equation for $H$ pick up extra contributions involving shear and spatial curvature. For Bianchi types I-VIII, where $R \leqslant 0$, the extra contributions to $H$ and $\dot{H}$ are positive and negative, respectively. As in the isotropic and spatially flat case it can be assumed without loss of generality that $H>0$, while $H$ is non-increasing and tends to a limit $H_{0} \geqslant 0$. A compactness argument as in previous sections shows that $\phi, \dot{\phi}, R$ and the square of the shear $\sigma_{a b} \sigma^{a b}$ tend to zero as $t \rightarrow \infty$. Beyond this point it must be expected that the asymptotics of solutions of different symmetry classes diverge, as they do in the case without a scalar field.

To the author's knowledge the only classes of homogeneous solutions of the Einstein equations coupled to a massive scalar field whose late-time asymptotics have been analysed further than in this paper are the Bianchi type I solutions and the isotropic solutions with nonvanishing spatial curvature. There is a heuristic discussion of the dynamics in these cases in 
$[1,2]$. One intuitive consideration for spacetimes containing only a massive scalar field similar to that concerning fluids at the end of section 4 follows from the equation $\mathrm{d} R / \mathrm{d} t=-2 H R$ which holds in a homogeneous, and isotropic model with non-zero curvature and the equation $\mathrm{d} / \mathrm{d} t\left(\sigma_{a b} \sigma^{a b}\right)=-6 H\left(\sigma_{a b} \sigma^{a b}\right)$ which holds in a Bianchi I spacetime. It suggests that in the latter case the influence of the shear at late times should be negligible, while in the former case the curvature should dominate. Indeed the claim in $[1,2]$ is that the late-time behaviour for negative curvature resembles that of the Milne model. Of course, in the case of positive curvature the solution might recollapse.

In general, it appears that it remains to obtain rigorous results for even some of the simplest models related to the massive scalar field. It would be interesting to see how the oscillations of the scalar field interact with those due to the gravitational field which arise in more complicated Bianchi types. For these Bianchi types averaging techniques are necessary to analyse the case without scalar field. $\mathrm{Cf}[7,12,17]$. Ideally a unified approach to all these problems should be developed.

\section{Acknowledgments}

I thank Spiros Cotsakis and John Miritzis for stimulating discussions.

\section{References}

[1] Belinskii V A, Grishchuk L P, Zeldovich Ya B and Khalatnikov I M 1986 Inflationary stages in cosmological models with a scalar field Sov. Phys. JETP 62 195-203

[2] Belinskii V A and Khalatnikov I M 1989 On the degree of generality of inflationary solutions of cosmological models with a scalar field Proc. 5th Marcel Grossmann Meeting ed D G Blair and M J Buckingham (Singapore: World Scientific)

[3] Bieli R 2006 Coupled quintessence and curvature-assisted inflation Class. Quantum Grav. 23 5983-95

[4] Kitada Y and Maeda K 1993 Cosmic no-hair theorem in homogeneous spacetimes: I. Bianchi models Class. Quantum Grav. $10703-34$

[5] Lee H 2005 The Einstein-Vlasov system with a scalar field Ann. H. Poincaré 6 687-723

[6] Mukhanov V 2005 Physical Foundations of Cosmology (Cambridge: Cambridge University Press)

[7] Nilsson U S, Hancock M J and Wainwright J 2000 Non-tilted Bianchi $\mathrm{VII}_{0}$ models — the radiation fluid Class. Quantum Grav. 17 3119-234

[8] Rendall A D 2002 Cosmological models and centre manifold theory Gen. Rel. Grav. 34 1277-94

[9] Rendall A D 2004 Accelerated cosmological expansion due to a scalar field whose potential has a positive lower bound Class. Quantum Grav. 21 2445-54

[10] Rendall A D 2005 Intermediate inflation and the slow roll approximation Class. Quantum Grav. 22 1655-66

[11] Rendall A D 2006 Dynamics of $k$-essence Class. Quantum Grav. 23 1557-69

[12] Ringström 2003 Future asymptotic expansions of Bianchi VIII vacuum metrics Class. Quantum Grav. 20 1943-90

[13] Rudin W 1987 Real and Complex Analysis (New York: McGraw-Hill)

[14] Starobinskii A A 1978 On a nonsingular isotropic cosmological model Sov. Astron. Lett. 4 82-4

[15] Starobinskii A A 1980 A new type of isotropic cosmological models without singularity Phys. Lett. B 91 99-102

[16] Starobinsky A A and Schmidt H-J 1987 On a general vacuum solution of fourth order gravity Class. Quantum Grav. 4 695-702

[17] Wainwright J, Hancock M J and Uggla C 1999 Asymptotic self-similarity breaking at late times in cosmology Class. Quantum Grav. 16 2577-98 